\title{
Long-term treatment of generalised myasthenia gravis with FK506 (tacrolimus)
}

\author{
T Konishi, Y Yoshiyama, M Takamori, T Saida, the Japanese FK506 MG Study Group*
}

J Neurol Neurosurg Psychiatry 2005;76:448-450. doi: 10.1136/jnnp.2004.042176

Efficacy and safety of long term use of FK506 (2-4.5 mg/ day) for a maximum of two years were evaluated in 12 patients with generalised myasthenia gravis (MG). At the end of the study, eight patients $(67 \%)$ showed improvement in either MG score or Activities in Daily Living score, and prednisolone dosage could be reduced in seven patients $(58 \%)$, with a mean reduction ratio of $37 \%$. Long term use of FK506 for MG can be more effective than short term administration, with no serious side effects.

M yasthenia gravis (MG), a T cell dependent chronic autoimmune disorder, is induced by sustained production of an antibody to nicotinic acetylcholine receptors (AChR) at the neuromuscular junction. ${ }^{1}$ FK506, an immunosuppressive drug discovered in Japan, ${ }^{2}$ acts on $\mathrm{T}$ helper cells to suppress production of various cytokines ${ }^{3}$ and thereby reduces the production of antibodies by B cells. We previously reported the results of a study of 16 weeks' treatment with FK506 (short term study) for generalised MG. Of 19 patients, nine patients (47\%) showed improvement in either MG or ADL (activities of daily living) score, and significant reduction of anti-AChR antibody titres and interleukin 2 production. ${ }^{4}$ Twelve patients (63\%) continued treatment with FK506 for a maximum of two years for evaluation of the efficacy and safety of its long term use. The remaining seven patients $(37 \%)$ did not continue the treatment because in three patients the treatment did not show efficacy and in four patients there were procedural difficulties at the treatment facilities ${ }^{4}$ (one patient moved away, one case in which long term study was not approved by an institutional review board, and two cases in which patient consent was not obtained).

\section{PATIENTS AND METHODS}

A total of 12 patients with generalised MG underwent long term treatment with FK506 for a maximum of two years at eight hospitals in Japan between May 1997 and May 2000, in accordance with the Declaration of Helsinki and the Good Clinical Practice guidelines. At entry, all patients were receiving prednisolone therapy at an ADL score of 2 or more and required additional immunosuppression to enhance clinical improvement or reduce the side effects of prednisolone, such as hyperlipidaemia, glaucoma, cataract, and osteoporosis. Patients receiving plasmapheresis $(n=5)$ or high dose intravenous $\gamma$-globulin therapy $(\mathrm{n}=1)$ entered this trial more than one month after therapy. Concurrent use of immunosuppressive agents other than prednisolone was prohibited during the trial. Azathioprine $(n=6)$, ciclosporin $(\mathrm{n}=2)$, and cyclophosphamide $(\mathrm{n}=2)$, if taken, were replaced by tacrolimus without a withdrawal period. Patients with disorders of the kidney, liver, pancreas, or heart were excluded. Clinical examination and laboratory tests were performed at about two month intervals following the initial 16 week study reported previously.
FK506 was given orally, once daily after dinner, at doses of 2-4.5 mg/day. Muscle strength was evaluated using a modified Besinger and Toyka MG score ${ }^{5}$ in order to assess symptoms of generalised MG. ADL score was assessed with a seven step rating scale:

- grade 0: no symptoms

- grade 1: presence of symptoms but no difficulty in carrying out daily activities, office work, or attending school

- grade 2: office work or school activities cannot be fully performed (including limitations caused by ocular symptoms)

- grade 3: office work, school activities, or outings are almost impossible, and disorders of phonation or swallowing are sometimes encountered

- grade 4: barely able to move around in a room or take care of oneself, and any disorder of phonation or swallowing is usually severe

- grade 5: bedridden or confined to a chair

- grade 6: requiring regular or occasional ventilatory assistance.

In all patients, the clinical assessment was performed at a fixed interval from the last administration of anticholinesterases to avoid modification by drugs. For MG score, a decrease of 3 or more points was considered an improvement, while an increase of 3 or more points was considered as worsening. For ADL, a change by 1 point or more was considered improvement/worsening.

Titres of anti-AChR antibodies were measured by the immunoprecipitation method using human AChR labelled with ${ }^{125} \mathrm{I}$ - $\alpha$-bungarotoxin as antigen. ${ }^{4}$

\section{RESULTS}

We treated 12 patients (three men, nine women; age range 28-59 years) with generalised MG. The duration of disease was 4-31 years. All patients had undergone thymectomy 3-28 years before entry into this study, and four patients (33\%; nos. 1, 4, 6, 11) had non-invasive thymoma. Patient numbers were carried over from the previous study. ${ }^{4}$

At the end of the long term study (after 88-104 weeks of treatment), two patients (nos. 4, 12) who had shown no change in the short term study showed improvement; one patient (no. 3) who had exhibited no change in the short term study had worsening of MG score. Overall, compared with scores at entry, the total MG score improved in five patients (nos. 1, 4, 12, 16, 17) and no change was observed in six (nos. 6, 8, 11, 13, 14, 15), (table 1). All patients had ADL scores of 2 or 3 at entry with half of the patients showing improvement at the end of the study: three patients $(25 \%$; nos. 1, 4, 17) exhibiting improvement by 2 points and three

Abbreviations: AChR, acetylcholine receptor; $A D L$, activities of daily living; $M G$, myasthenia gravis 
Table 1 Summary of patient data

\begin{tabular}{|c|c|c|c|c|c|c|c|c|c|c|c|c|c|}
\hline \multirow[b]{2}{*}{ Patient no.* } & \multirow[b]{2}{*}{ Age } & \multirow[b]{2}{*}{ Sex } & \multirow{2}{*}{$\begin{array}{l}\text { Duration of } \\
\text { disease (years) }\end{array}$} & \multirow{2}{*}{$\begin{array}{l}\text { FK506 dose } \\
\text { (mg/day) }\end{array}$} & \multicolumn{2}{|c|}{ Total MG score } & \multicolumn{2}{|c|}{ ADL score } & \multicolumn{2}{|c|}{$\begin{array}{l}\text { Prednisolone daily } \\
\text { dose (mg) }\end{array}$} & \multicolumn{2}{|c|}{$\begin{array}{l}\text { Antibody titre } \\
\text { (nM) }\end{array}$} & \multirow[b]{2}{*}{ Previous therapy } \\
\hline & & & & & Entry & Exit & Entry & Exit & Entry & Exit & Entry & Exit & \\
\hline 1 & 41 & $\mathrm{~F}$ & 15 & $2-3$ & 14 & 3 & 3 & 1 & $50 / 20+$ & $25 / 5 \dagger$ & 44 & 22 & \\
\hline 3 & 59 & $\mathrm{~F}$ & 5 & $3-4.5$ & 9 & 12 & 3 & 3 & 5 & 5 & 3.9 & 1.8 & AZP, CyA, CPA, PE \\
\hline 4 & 56 & $M$ & 9 & $3-4$ & 5 & 1 & 3 & 1 & $17.5 / 0+$ & $10 / 0 \dagger$ & 38 & 12 & \\
\hline 6 & 50 & $M$ & 7 & 3 & 0 & 1 & 2 & 2 & $15 / 0 \dagger$ & $7.5 / 0 \dagger$ & 7.8 & 2.7 & $A Z P, P E$ \\
\hline 8 & 49 & $\mathrm{~F}$ & 31 & 3 & 13 & 11 & 3 & 3 & $20 / 0+$ & $20 / 0+$ & 69 & 65 & CyA \\
\hline 11 & 46 & $\mathrm{~F}$ & 8 & 3 & 1 & 0 & 2 & 2 & $25 / 0+$ & $12.5 / 0 \dagger$ & 23 & 19 & \\
\hline 12 & 28 & $\mathrm{~F}$ & 4 & 3 & 6 & 2 & 2 & 2 & $25 / 0+$ & $25 / 0 \dagger$ & 11 & 12 & \\
\hline 13 & 51 & $\mathrm{~F}$ & 12 & 3 & 13 & 11 & 2 & 1 & 10 & 10 & 3.2 & 2.1 & AZP \\
\hline 14 & 52 & $\mathrm{~F}$ & 29 & 3 & 5 & 6 & 2 & 1 & $15 / 5+$ & $15 / 0+$ & $<0.2$ & $<0.2$ & AZP , CPA, PE, IVlg \\
\hline 15 & 50 & $\mathrm{~F}$ & 18 & 3 & 2 & 1 & 2 & 1 & $40 / 0+$ & $35 / 0+$ & 0.8 & 0.3 & AZP \\
\hline 16 & 38 & $\mathrm{~F}$ & 4 & 3 & 16 & 4 & 2 & 2 & 20 & 20 & 11 & 4.2 & $A Z P, P E$ \\
\hline 17 & 47 & $M$ & 4 & 3 & 6 & 0 & 2 & 0 & $30 / 0+$ & $27.5 / 0 \dagger$ & 41 & 21 & PE \\
\hline
\end{tabular}

Patient nos. 1, 3, 4, 6, 8, 11, 12: 104 weeks treatment; no. 13: 96 weeks treatment; nos. 14, 15, 16, 17: 88 weeks treatment

*Patient numbers carried over from previous study. ${ }^{4}$

†Alternate day therapy.

AZP, azathioprine; CyA, ciclosporin; CPA, cyclophosphamide; PE, plasmapheresis; IVlg, high dose intravenous $\gamma$-globulin

patients showing improvement by 1 point (nos. 13, 14, 15). Thus 8/12 (67\%) patients showed improvement in either MG or ADL score. In addition, prednisolone dosage could be reduced with a mean reduction rate of $37 \%(8.3-57.0 \%)$ in seven patients, of whom five had improvement in ADL score and two had an unchanged ADL score. No significant relation was observed between anti-AChR antibody titre, presence of thymoma, duration of disease and MG or ADL improvement. The antibody titre reduced in 10/12 (83\%) patients (from a mean value of $21.1 \mathrm{nM}$ to $13.6 \mathrm{nM}$; Wilcoxon's signed rank test, $\mathrm{p}<0.01$ ). One patient (no. 14) was seronegative, but was diagnosed as having MG on the basis of clinical presentation, positive edrophonium chloride (Tensilon) test, decremental response on repetitive nerve stimulation, and beneficial response to anticholinesterase medication.

In eight patients, 22 side effects were observed. One patient suffered from severe headache and eye pain that required discontinuation of FK506 therapy for 48 days but was subsequently able to be treated again with the same agent. Other side effects were minor and FK506 therapy could be continued without any specific measures for those side effects. An increase in neutrophil count and a decrease in lymphocyte count were observed in four patients $(33 \%)$. Infection as a side effect was not observed.

\section{DISCUSSION}

When FK506 was given for a maximum of two years to 12 patients with generalised MG, total MG or ADL score improved in eight patients $(67 \%)$. This response rate is higher than that observed in our previous short term study $(47 \%){ }^{4}$ Furthermore, in three patients the ADL score improved by 2 or more (none in the short term study) and prednisolone dosage was successfully reduced in seven patients (only three in the short term study). These findings strongly indicate the usefulness of long term treatment with FK506 and its advantages over short term treatment. Ciclosporin $^{1}$ and $\mathrm{FK} 56^{4}$ are characterised by more rapid onset of efficacy against MG than other immunosuppressants. However, given the results of the current long term study, including cases of late onset of efficacy (after 26 weeks or more of treatment), FK506 appears to have further improving potential with long term use. Azathioprine is most useful in patients with MG in whom corticosteroids are contraindicated, in those with an insufficient response to steroids, or as an adjunct to permit a reduction in the steroid dose. ${ }^{1}$ A randomised double blind trial of prednisolone alone or with azathioprine in MG confirmed its usefulness. ${ }^{6}$ Azathioprine takes one to two years to have its maximum effect in MG. ${ }^{1}$

In a study performed in the USA and Europe on long term use of ciclosporin over two years in nine patients with severe MG, clinical response was obtained in seven patients and corticosteroid dosage reduction by half or more was possible in seven patients. ${ }^{7}$ However, reduction in anti-AchR antibody titre was observed in only $2 / 9(22 \%)$ patients. In another retrospective study of ciclosporin for an average of 3.5 years, 55/57 (96\%) patients showed clinical improvement with 57\% reduction in antibody level in nine patients and corticosteroids were discontinued or decreased in 36/38 (95\%) patients who were taking these. ${ }^{8}$ Owing to differences in dosage, no simple comparison can be made in terms of response rate and other variables between the above studies and the present FK506 long term study. Nevertheless, long term FK506 therapy resulted in greater reduction in antibody titres (35.5\% reduction in 10 patients $(83 \%))$. Ciclosporin had more rapid onset of efficacy than azathioprine ${ }^{1}$ and FK506 was significantly effective for lower limb muscle strength two weeks after administration in a short term clinical trial. ${ }^{4}$

Regarding adverse events, long term ciclosporin therapy increased serum creatinine levels in $8 / 9(89 \%)^{7}$ and $16 / 57$ (28\%) patients, ${ }^{8}$ whereas long term FK506 therapy induced no increase in serum creatinine levels. One of the side effects observed in FK506 treatment of MG is diabetes mellitus. ${ }^{9}$ The diabetogenic action of FK506 on pancreatic $\beta$ cells is due to transcriptional inhibition of insulin. ${ }^{10}$ No diabetogenic adverse effects were observed in the 12 patients treated with FK506 in this long term study, but it has been reported that diabetes mellitus exacerbated by FK506 can be relieved by dose reduction. ${ }^{9}$

In summary, long term use of FK506 in low doses $(2-4.5 \mathrm{mg} /$ day) is safe and effective in patients with generalised MG in association with prednisolone reduction. In the future, a more extensive and blinded study may be needed to establish the real efficiency of FK506, which would be another option of treatment of MG.

\section{Authors' affiliations}

T Konishi, T Saida, Department of Neurology, Utano National Hospital, Kyoto, Japan

Y Yoshiyama, Department of Neurology, Chiba University School of Medicine, Chiba, Japan

M Takamori, Neurological Center, Kanazawa-Nishi Hospital, Kanazawa, Japan 
This study was supported by a grant from Fujisawa Pharmaceutical Co, Ltd, Osaka, Japan.

Competing interests: none declared

*Other participating members of the Japanese FK506 MG Study were: E Mukai (National Nagoya Hospital); G Sobue (Nagoya University School of Medicine); F Kanda (Kobe University School of Medicine); J-I Kira (Kyushu University School of Medicine); T Nakamura (Nagasaki University School of Medicine).

Presented in part at the EFNS Congress in Vienna, Austria, October 2002.

Correspondence to: DrT Konishi, Department of Neurology, Utano National Hospital, 8, Ondoyama-cho, Narutaki, Kyoto 616-8255,

Japan; konishi@unh.hosp.go.jp

Received 4 April 2004

In revised form 7 July 2004

Accepted 15 July 2004

\section{REFERENCES}

1 Drachman DB. Myasthenia gravis. N Engl J Med 1994;330:1797-810.

2 Kino T, Hatanaka H, Hashimoto M, et al. FK-506, a novel immunosuppressant isolated from a Streptomyces. I. Fermentation, isolation, and physicochemical and biological characteristics. J Antibiot (Tokyo) 1987;40:1249-55.

3 Schreiber SL, Crabtree GR. The mechanism of action of cyclosporin A and FK506. Immunol Today 1992;13:136-42.

4 Konishi T, Yoshiyama Y, Takamori M, et al. A clinical study of FK506 in patients with myasthenia gravis. Muscle Nerve 2003;28:570-4.

5 Besinger UA, Toyka KV, Hömberg M, et al. Myasthenia gravis: long-term correlation of binding and bungarotoxin blocking antibodies against acetylcholine receptors with changes in disease severity. Neurology 1983;33:1316-21.

6 Palace J, Newsom-Davis J, Lecky B. A randomized double-blind trial of prednisolone alone or with azathioprine in myasthenia gravis. Neurology 1998;50:1778-3.

7 Bonifati DM, Angelini C. Long-term cyclosporine treatment in a group of severe myasthenia gravis patients. J Neurol 1997;244:542-7.

8 Ciafaloni E, Nikhar NK, Massey JM, et al. Retrospective analysis of the use of cyclosporine in myasthenia gravis. Neurology 2000;55:448-50.

9 Evoli A, Schino CD, Marsili F, et al. Successful treatment of myasthenia gravis with tacrolimus. Muscle Nerve 2002;25:111-14.

10 Tamura K, Fujimura T, Tsutsumi T, et al. Transcriptional inhibition of insulin by FK506 and possible involvement of FK506 binding protein-12 in pancreatic $\beta$ cell. Transplantation 1995;59:1606-13. 